TRANSACTIONS OF THE

AMERICAN MATHEMATICAL SOCIETY

Volume 343, Number 1, May 1994

\title{
THE HEXAGONAL PACKING LEMMA AND THE RODIN SULLIVAN CONJECTURE
}

\author{
DOV AHARONOV
}

\begin{abstract}
The Hexagonal Packing Lemma of Rodin and Sullivan [6] states that $s_{n} \rightarrow 0$ as $n \rightarrow \infty$. Rodin and Sullivan conjectured that $s_{n}=O(1 / n)$. This has been proved by Z-Xu He [2]. Earlier, the present author proved the conjecture under some additional restrictions [1].

In the following we are able to remove these restrictions, and thus give an alternative proof of the RS conjecture. The proof is based on our previous article [1]. It is completely different from the proof of $\mathrm{He}$, and it is mainly based on discrete potential theory, as developed by Rodin for the hexagonal case [4].
\end{abstract}

\section{INTRODUCTION}

The Hexagonal Packing Lemma plays an important role in the proof of Rodin and Sullivan of Thurston's conjecture that his scheme converges to the Riemann mapping.

It was suggested by Rodin and Sullivan $[4,5,6]$ that $s_{n}=O(1 / n)$. This property is important for investigation of the behavior of the derivatives. More specifically, $s_{n}=O(1 / n)$ implies the convergence of the "circle function" $r_{\varepsilon}$ to the modulus of the derivative of the Riemann mapping function $[5,6]$. [2]).

It is easy to show that one cannot get anything better than $s_{n}=O(1 / n)$ (cf.

In what follows, we prove the RS conjecture. The proof is based on our previous paper [1]. The main additional tool in the present work is an assertion of a "mean convergence" which is, in a way, a form of discrete $L^{2}$ convergence. This "mean convergence" allows us to remove the restriction in [1] and thus get the full result by an induction process.

We use freely the standard notations and definitions introduced by the researchers working in the area (cf. $[4,5,6])$. In particular, $\mathrm{HCP}_{N}$ denotes the first $N$ generations of the regular hexagonal circle packing, with all radii equal to 1 . $\mathbf{H C P}_{N}^{\prime}$ denotes a circle packing that is combinatorially isomorphic to $\mathrm{HCP}_{N}$.

In addition $H L(h)$ is the hexagonal lattice of mesh $h . H L(h, N)$ denotes the subset of $H L(h)$ consisting of lattice points of generations $\leq N$ (see [4]).

Received by the editors March 12, 1992, and in revised form, May 12, 1992.

1991 Mathematics Subject Classification. Primary 30C85; Secondary 30C60, 31A05, 31C20. 
Also in the following we shall use the notation $\overline{s_{N}}$ instead of $s_{N}$ in order to emphasize the fact that the additional restriction (2.1) is added.

\section{Preliminary Results}

We first state our main result in [1] in a slightly different form, which is more suitable for our purposes.

Theorem 2.1 [1]. Let $\mathrm{HCP}_{N}^{\prime}$ be a circle packing combinatorially isomorphic to $\mathrm{HCP}_{N}$. Then there exist two numerical constants $\rho>0, A>0$ with the following property: If

$$
\exp (-3 \rho) \leq r \leq \exp (3 \rho)
$$

for each of the radii of the circles in $\mathrm{HCP}_{N}^{\prime}$, then for any natural $N$

$$
\overline{s_{N}}<A / N \text {. }
$$

Also:

$$
\rho \leq \min (\log 2, A) .
$$

It is worthwhile to note that the above theorem is, in fact, a statement on a certain class of functions. More specifically, if both $u$ and $1 / u$ are subharmonic discrete functions on $H L(1, N)$ and if, in addition, the " $\rho$ condition"

$$
\exp (-3 \rho) \leq u \leq \exp (3 \rho)
$$

is satisfied, then (2.2) holds. In this more general setting, the above theorem is sharp, in the sense that the " $\rho$ condition" cannot be removed.

Indeed, consider the subharmonic function

$$
u=u(x, y)=\exp (3 \rho x / N)
$$

defined on $H L(1, N)$. It is an easy matter to show that both $u$ and $1 / u$ are subharmonic discrete functions. If $x$ is an integer and $-N \leq x \leq N, u$ satisfies the " $\rho$ condition" $(2.4)$ on $H L(1, N)$. Hence, it follows by Theorem 2.1 that $u(1) / u(0)=\exp (3 \rho / N)=O(1 / N)$. On the other hand, it is clear that the " $\rho$ condition" cannot be omitted since, if instead of $(2.5)$, one considers the function $u(x, y)=\exp (M x / N)$, then $u$ and $1 / u$ are still subharmonic, but $u(1) / u(0)$ depends on $M$, and thus (2.2) cannot be satisfied for an arbitrary large $M$.

It is therefore clear that in order to prove the RS conjecture without the restriction of the " $\rho$ condition", one needs additional information arising from the particular geometry of the present situation, in addition to the property of discrete subharmonicity of the "circle function" $r$ and its reciprocal $1 / r$.

In the sequel we consider, for some positive integer $l$, the six configurations $\mathrm{HCP}_{N}, \mathrm{HCP}_{2 N}, \mathrm{HCP}_{l N}, \mathrm{HCP}_{N}^{\prime}, \mathrm{HCP}_{2 N}^{\prime}, \mathrm{HCP}_{l N}^{\prime}$.

We now introduce some additional notation: $\mathrm{HCP}_{N, q}$ will denote the regular hexagonal configuration, but with radius $q$ and not $1 . f_{l N}$ denotes the $k$-qc ( $k$ quasi conformal) mapping from the carrier $[5,6]$ of $\mathrm{HCP}_{l N, N^{-1}}$ onto the carrier of $\mathrm{HCP}_{l N}^{\prime}$.

Later on we will say occassionally that $f_{l N}$ maps $\mathrm{HCP}_{l N, N^{-1}}$ onto $\mathrm{HCP}_{l N}^{\prime}$, in those places where there is no danger of ambiguity. We recall a few important facts $[5,6]$ about the method of constructing functions similar to the function $f_{l N}$. The center of each circle is mapped onto the center of the image circle by 
the isomorphic correspondence. The map $f_{l N}$ is then extended by the barycentric coordinates. Using the Ring Lemma [6] one knows that there is a uniform $k$ which is independent of $N$ and $l$, s.t. the constructed map is $k$-qc. We also note that $f_{l N}(0)=0$ is not a restriction and this will be assumed throughout.

Later we consider the function $g_{l N}=f_{l N} / f_{l N}(1)$ with the normalization

$$
g_{l N}(0)=0, \quad g_{l N}(1)=1 .
$$

Also, the function $g_{l N}$ is $k$-qc, with the same $k$ as $f_{l N}$.

As $f_{l N}$ maps $\mathrm{HCP}_{l N, N^{-1}}$ onto $\mathrm{HCP}_{l N}^{\prime}$, the function $g_{l N}$ maps $\mathrm{HCP}_{l N, N^{-1}}$ onto $\mathrm{HCP}_{l N}^{*}$ which denotes the configuration $\mathrm{HCP}_{l N}^{\prime}$ "divided" by $f_{l N}(1)$. Of course, each of the radii of circles in $\mathrm{HCP}_{l N}^{\prime}$ is divided by $f_{l N}(1)$, to get the corresponding value of the radius of the image circle in $\mathrm{HCP}_{l N}^{*}$.

The above normalization (2.6) is needed for constructing a normal family of $k$-qc mappings. In any case, there is a simple invariance property which is of importance: since $g_{l N}=f_{l N} / f_{l N}(1)$, and the relation is only an expansion (or contraction) by $f_{l N}(1)^{-1}$, the ratio between two radii is not changed. Later on we will use this invariance property, while dealing with statements about ratio of two radii.

We now recall a known result that will be needed to establish the "normal family" property of the class $\left\{g_{l N}\right\}$. For the convenience of the reader we bring the proof of this result.

Lemma 2.1. Let $G$ be a family of $k$-qc mappings in a plane domain $D$. Let a, $b$ be two distinct points in $D$, s.t. $f(a)=a, f(b)=b$, for each $f \in G$. Also assume that for each $f \in G$, a certain fixed value $($ say $\infty)$ is omitted. Then $G$ is a normal family.

Proof. Consider the class $G$ restricted to $D \backslash\{a\} . G$ is a family of $k$-qc mappings omitting the two values $\{a, \infty\}$ in $D \backslash\{a\}$. Hence, $G$ is a normal family in $D \backslash\{a\}$ [3, p. 73]. Thus, from a given sequence $\left\{f_{n}\right\}$ in $G$, we can choose a subsequence $\left\{f_{n_{p}}\right\}$ s.t. $f_{n_{p}}$ converges uniformly on each compact subset of $D \backslash\{a\}$. Now, consider the sequence $\left\{f_{n_{p}}\right\}$ restricted to $D \backslash\{b\}$. This set of functions omit the two values $\{b, \infty\}$. Thus, again, we can find a subsequence, say $\left\{f_{n_{p}}^{s}\right\} \subset\left\{f_{n_{p}}\right\}$ that converges uniformly on each compact subdomain of $D \backslash\{b\}$. Hence $\left\{f_{n_{p}}^{s}\right\}$, which is a subsequence of $\left\{f_{n_{p}}\right\}$ by construction, converges uniformly on each compact subdomain of $D$, and the proof is complete.

Note that the limit function is $k$-qc mapping with the same $k$, and it cannot be a constant on $D$, since the family has two different fixed points.

\section{THE "MEAN CONVERGENCE" PROPERTY}

Lemma 3.1. Let $\left\{g_{l N}\right\}$ be defined as above with the normalization (2.6). $g_{l N}$ maps $\mathrm{HCP}_{l N, N^{-1}}$ on $\mathrm{HCP}_{l N}^{*}$. Then, given $\varepsilon>0$ and a compact domain $D \subset \mathbb{C}$, there exist $N_{0}=N(\varepsilon, D), l_{0}=l(\varepsilon, D)$ s.t.

$$
\left|g_{l N}(z)-z\right|<\varepsilon, \quad \text { if } z \in D, N \geq N_{0}, l \geq l_{0} .
$$

Also, $N_{0}, l_{0}$ do not depend on the particular sequence $\left\{g_{l N}\right\}$.

Proof. By Lemma 2.1 applied for the family $\left\{g_{l N}\right\}$, it is clear that $\left\{g_{l N}\right\}$ form a normal family for each disc of radius $R>0$ provided $l$ is large enough. 
This follows at once from the fact that the domain of definition of $g_{l N}$ is $\mathrm{HCP}_{l N, N^{-1}}$, and it contains a disc of radius $l$, as can be easily seen.

If Lemma 3.1 is not valid for some $D$, there exist an $\varepsilon>0$ and two sequences $\left\{l_{j}\right\}_{j=1}^{\infty},\left\{N_{j}\right\}_{j=1}^{\infty}$ s.t. $l_{j} \rightarrow \infty, N_{j} \rightarrow \infty$ as $j \rightarrow \infty$ and a sequence $\left\{g_{l_{j} N_{j}}\right\}$ for which $\max _{z \in D}\left|g_{l_{j} N_{j}}(z)-z\right| \geq \varepsilon$. By taking subsequences and using the standard diagonal process, we may assume that $g_{l_{j} N_{j}}(z)$ converges uniformly in any compact subdomain of $\mathbb{C}$. Also, since $s_{n} \rightarrow 0$ as $n \rightarrow \infty$, it follows that $g_{l_{j} N_{j}}$ tends to a univalent analytic mapping. (We omit the details of the proof of this fact. Instead of the reader is referred to [6] where an almost identical situation occurs, and the limiting process is used to show the convergence of the "circle functions" $r_{\varepsilon}(z)$ to the Riemann map of a given plane domain onto the unit disc). Continuing with our argument, we can conclude now that the limit function must be a univalent analytic map on $\mathbb{C}$. Such a map is necessarily linear, and must be of the form $\alpha z+\beta$, for some constants $\alpha, \beta$. But $\{0,1\}$ are two fixed points for each $g_{l_{j} N_{j}}$. Hence, the same is true for the limit function. We thus end up with the conclusion that the limiting function must be the identity map. Thus, $g_{l_{j} N_{j}}$ converges uniformly in any compact subdomain of $\mathbb{C}$ to the identity function $I(z) \equiv z$. This contradicts the condition $\max \left|g_{l_{j} N_{j}}(z)-z\right| \geq \varepsilon$ and the proof is complete.

We now state another variant of Lemma 3.1, in a form that will be more convenient for applications. For this aim we take a particular $D$ in Lemma 3.1 , namely, the disc $\{z,|z| \leq 5\}$. Also, consider the function $\varphi_{l N}(z)=$ $N g_{l N}(z / N)$ which maps $\mathrm{HCP}_{l N}$ on the configuration $\mathrm{HCP}_{l N}^{*}$ "multiplied" by $N$. We denote this "expanded" domain ty $\mathrm{HCP}_{l N}^{* *}$. It is also clear that

$$
\varphi_{l N}(0)=0, \quad \varphi_{l N}(N)=N \text {. }
$$

We now have

Lemma 3.2. Let $\left\{\varphi_{l N}\right\}$ be defined as above with the normalization (3.2). $\varphi_{l N}$ maps $\mathrm{HCP}_{l N}$ on $\mathrm{HCP}_{l N}^{* *}$. Then, given $\varepsilon>0$ there exist $N_{0}=N(\varepsilon), l_{0}=l(\varepsilon)$, s.t. for $N \geq N_{0}, l_{0} \geq l_{0}$,

$$
\left|\varphi_{l N}(z)-z\right|<\varepsilon N, \quad \text { if }|z| \leq 5 N .
$$

Also, $N_{0}, l_{0}$ do not depend on the particular choice of $\left\{\varphi_{I N}\right\}$.

The proof follows at once from Lemma 3.1, by taking $D$ as the disc $|z| \leq 5$ and using $\varphi_{l N}=N g_{l N}(z / N)$.

We shall need also the following elementary trigonometric assertion.

Lemma 3.3 (see Figure 1). Let $\left\{K_{j}\right\}_{1}^{3}$ be three mutually tangent discs with disjoint interior. Denote, further, the radii of these discs by $\left\{r_{j}\right\}_{1}^{3}$, and their centers by $\left\{a_{j}\right\}_{1}^{3}$, respectively. Let the triangle with the three vertices $\left\{a_{j}\right\}_{1}^{3}$ be denoted by $T$, and its area by $S(T)$. Denote by $S$ the area of those parts of the discs covered by $T$. Then, given any $\varepsilon>0$, we may find $\delta=\delta(\varepsilon)$, s.t. if $1-\delta<r_{j} / r_{k}<1+\delta,\{j, k\} \subset\{1,2,3\}$ then

$$
\frac{\pi \sqrt{3}}{6}(1-\varepsilon)<S / S(T)<\frac{\pi \sqrt{3}}{6}(1+\varepsilon) .
$$



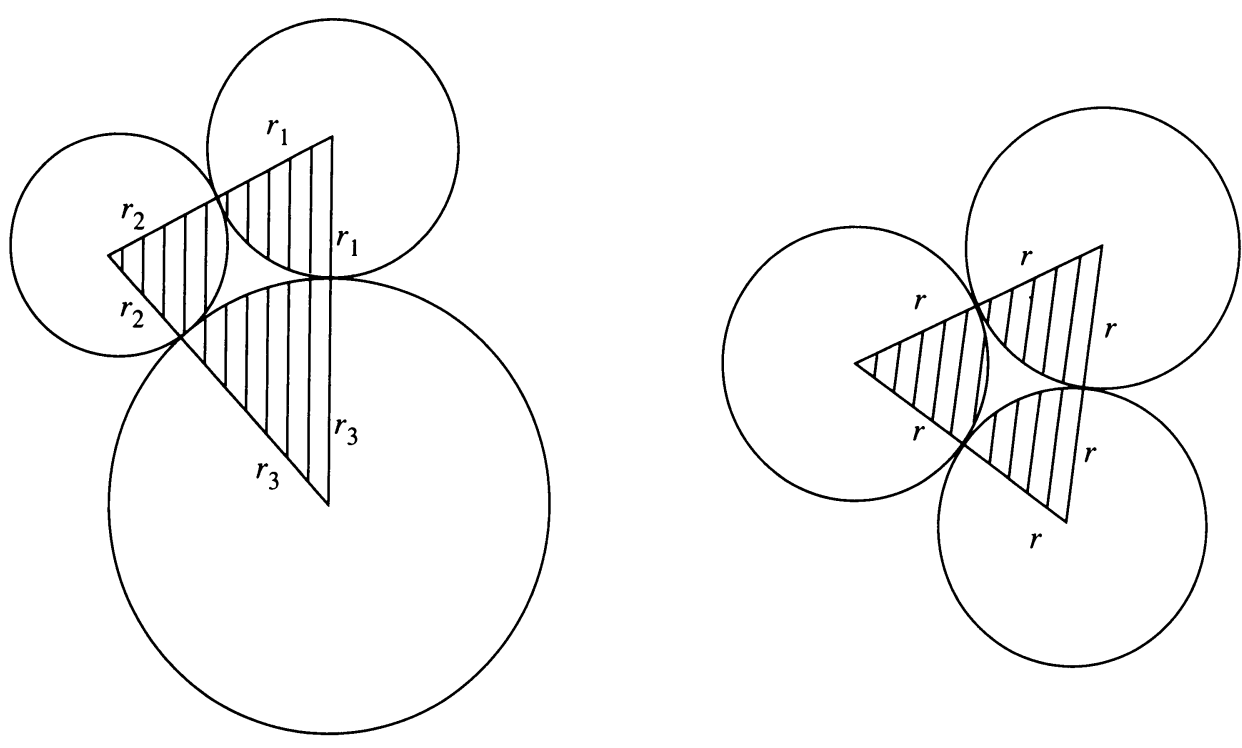

FIGURE 1

Proof. For the regular case we have $S / S(T)=\pi \sqrt{3} / 6$, as follows easily by an elementary argument. To get Lemma 3.3 , we can either make a simple calculation, or use an obvious continuity argument.

Our aim now is to present the main result in this section. In what follows, consider the configurations $\mathrm{HCP}_{N}, \mathrm{HCP}_{2 N}, \mathrm{HCP}_{l N}$. Also, $f_{l N}, g_{l N}, \varphi_{l N}$ are the functions constructed above. Hence $\varphi_{l N}$ satisfies (3.2) and maps $\mathrm{HCP}_{l N}$ onto $\mathrm{HCP}_{l N}^{* *}$. We have

Theorem 3.1 (Discrete "mean convergence"). Given $\varepsilon>0$ and any sequence $\left\{\varphi_{l N}\right\}$ defined as above, there exist $N_{1}=N_{1}(\varepsilon), l_{1}=l_{1}(\varepsilon)$, s.t.

$$
\sum_{j=N+1}^{2 N} \sum_{k=1}^{6 j}\left(1-r_{j k}\right)^{2}<\varepsilon^{2} N^{2}
$$

for $N \geq N_{1}, l \geq l_{1}$, where the $\left\{r_{j k}\right\}$ are the radii of the circles in the configuration $\mathrm{HCP}_{l N}^{* *}$. Also, $N_{1}, l_{1}$ do not depend on the particular sequence $\left\{\varphi_{l N}\right\}$.

Proof. We first emphasize the fact that $N_{1}, l_{1}$ depend only on $\varepsilon$, and not on the particular sequence $\left\{\varphi_{l N}\right\}$. Also, we point out that the disc $\{z,|z| \leq 5 N\}$, appearing in (3.3), contains the configuration $\mathrm{HCP}_{2 N}$.

Our aim now is to compare the map $\varphi_{l N}$ and the identity map $I(z) \equiv z$. For $I(z)$ the left-hand side of (3.5) is obviously zero since all the radii are equal to one. For the map $\varphi_{l N}$ we rewrite the left-hand side of (3.5):

$$
\sum_{N+1}^{2 N} \sum_{1}^{6 j}\left(1-r_{j k}\right)^{2}=\left(\sum_{N+1}^{2 N} \sum_{1}^{6 j} r_{j k}^{2}-\sum_{N+1}^{2 N} \sum_{1}^{6 j} 1\right)+2\left(\sum_{N+1}^{2 N} \sum_{1}^{6 j} 1-\sum_{N+1}^{2 N} \sum_{1}^{6 j} r_{j k}\right) .
$$

Next, we plan to show that each of the two expressions on the right-hand side of (3.6) is $o\left(N^{2}\right)$. This will be done with the aid of Lemmas 3.2 and 3.3. 
To estimate the expression $2\left(\sum \sum 1-\sum \sum r_{j k}\right)$ we use Lemma 3.2, taking $\varepsilon^{2} / 24$ instead of $\varepsilon$. Hence, from (3.3), and a simple geometric consideration (3.7)

$$
\sum_{k=1}^{6 j} r_{j k}>6 j-\frac{1}{4} \varepsilon^{2} N, \quad N+1 \leq j \leq 2 N, \quad \forall N \geq N_{0}\left(\frac{\varepsilon^{2}}{24}\right), \quad \forall l \geq l_{0}\left(\frac{\varepsilon^{2}}{24}\right),
$$

which implies

$$
\sum_{j=N+1}^{2 N} \sum_{k=1}^{6 j} r_{j k}>\sum_{j=N+1}^{2 N}\left(\sum_{k=1}^{6 j} 1-\frac{\varepsilon^{2}}{4} N\right)
$$

and

$$
\begin{aligned}
2\left(\sum_{j=N+1}^{2 N} \sum_{k=1}^{6 j} 1-\sum_{j=N+1}^{2 N} \sum_{k=1}^{6 j} r_{j k}\right)<\frac{\varepsilon^{2}}{2} N^{2}, \\
\forall N \geq N_{0}\left(\frac{\varepsilon^{2}}{24}\right), \forall l \geq l_{0}\left(\frac{\varepsilon^{2}}{24}\right) .
\end{aligned}
$$

For the estimation of the other expression appearing on the right-hand side of (3.6), first observe that $\pi \sum_{j=N+1}^{2 N} \sum_{k=1}^{6 j} r_{j k}^{2}$ is the area of the set of discs belonging to $\mathrm{HCP}_{2 N}^{* *} \backslash \mathrm{HCP}_{N}^{* *}$. Similarly, $\pi \sum_{j=N+1}^{2 N} \sum_{k=1}^{6 j} 1^{2}$ is the area of the set of discs belonging to $\mathrm{HCP}_{2 N} \backslash \mathrm{HCP}_{N}$. Next, compare the area of the two carriers of $\mathrm{HCP}_{2 N} \backslash \mathrm{HCP}_{N}$ and $\mathrm{HCP}_{2 N}^{* *} \backslash \mathrm{HCP}_{N}^{* *}$. We now prefer to proceed in a less formal way and leave aside part of the somewhat long (but elementary) computational details.

Using, once more, Lemma 3.2, we have for $N, l \rightarrow \infty$

$$
\begin{array}{r}
\mid \text { area of carrier }\left(\mathrm{HCP}_{2 N}^{* *} \backslash \mathrm{HCP}_{N}^{* *}\right) \\
- \text { area of carrier }\left(\mathrm{HCP}_{2 N} \backslash \mathrm{HCP}_{N}\right) \mid=o\left(N^{2}\right) .
\end{array}
$$

Also note that each one of the circles in $\mathrm{HCP}_{2 N}^{* *}$ is surrounded by a subpacking of $\mathrm{HCP}_{l N}^{* *}$ that contains at least $(l-2) N$ generations. By taking $(l-2) N$ large enough, we can make $s_{(l-2) N}$ as small as we please. (Indeed, this follows from the RS theorem, i.e., $s_{n} \rightarrow 0$ for $n \rightarrow \infty$.) Our aim is now to combine this fact with Lemma 3.3. For this, take $K_{j_{1}}, K_{j_{2}}, K_{j_{3}}$ (in place of the $K_{j}$ ) that are mutually tangent and belong to the configuration $\mathrm{HCP}_{2 N}^{* *} \backslash \mathrm{HCP}_{N}^{* *}$. Using what has been said about the convergence of $s_{(l-2) / N}$ to zero, it is clear from (3.4) that

$$
S_{j_{1} j_{2} j_{3}}=S\left(T_{j_{1} j_{2} j_{3}}\right) \cdot \frac{\pi \sqrt{3}}{6}(1+o(1))
$$

with an obvious meaning of the notations.

Note that $o(1)=O\left(s_{(l-2) N}\right)$ is uniform for all triplets $\left\{K_{j_{1}}, K_{j_{2}}, K_{j_{3}}\right\}$ belonging to $\mathrm{HCP}_{2 N}^{* *} \backslash \mathrm{HCP}_{N}^{* *}$. Also note that the number of triangles in the carrier of $\mathrm{HCP}_{2 N}^{* *} \backslash \mathrm{HCP}_{N}^{* *}$ is $O\left(N^{2}\right)$. On summation we have from (3.10):

(3.11) $\pi \sum_{j=N+1}^{2 N} \sum_{k=1}^{6 j} r_{j k}^{2}=$ area of carrier $\left(\mathrm{HCP}_{2 N}^{* *} \backslash \mathrm{HCP}_{N}^{* *}\right) \cdot \frac{\pi \sqrt{3}}{6}(1+o(1))$.

$$
\pi \sum_{j=N+1}^{2 N} \sum_{k=1}^{6 j} 1^{2}=\text { area of carrier }\left(\mathrm{HCP}_{2 N} \backslash \mathrm{HCP}_{N}\right) \cdot \frac{\pi \sqrt{3}}{6}(1+o(1)) .
$$


From (3.9), (3.11), (3.12),

$$
\pi \sum_{j=N+1}^{2 N} \sum_{k=1}^{6 j} r_{j k}^{2}-\pi \sum_{j=N+1}^{2 N} \sum_{k=1}^{6 j} 1^{2}=o\left(N^{2}\right) .
$$

Writing this "quantitatively" we get

For any $\varepsilon>0$ there exist $\bar{N}_{0}=\bar{N}_{0}(\varepsilon), \bar{l}_{0}=\bar{l}_{0}(\varepsilon)$ s.t. if $N \geq \bar{N}_{0}, l \geq \bar{l}_{0}$

$$
2\left(\sum_{j=N+1}^{2 N} \sum_{k=1}^{6 j} r_{j k}^{2}-\sum_{j=N+1}^{2 N} \sum_{k=1}^{6 j} 1^{2}\right)<\frac{\varepsilon^{2}}{2} N^{2} .
$$

From (3.8) and (3.13) the proof of Theorem 3.1 follows by taking $N_{1}=N_{1}(\varepsilon)=$ $\max \left(N_{0}, \bar{N}_{0}\right), l_{1}=l_{1}(\varepsilon)=\max \left(l_{0}, \bar{l}_{0}\right)$.

\section{CONNECTION BETWEen THE "MEAN CONVERGENCE" AND THE " $\rho$ CONDITION"}

In the foliowing, whenever $\rho$ and $A$ are mentioned, they are the specific numerical constants appearing in $\S 2$. We further denote

$$
\varepsilon_{1}=\varepsilon(\rho, A)=\rho^{2} / 4 A \text {. }
$$

Hereafter, $\varepsilon_{1}$ will mean this specific numerical constant.

From (3.5) we have

$$
\sum_{j=N+1}^{2 N} \sum_{k=1}^{6 j}\left(1-r_{j k}\right)^{2}<\varepsilon_{1}^{2} N^{2}
$$

provided $N \geq N_{1}\left(\varepsilon_{1}\right), l \geq l_{1}\left(\varepsilon_{1}\right)$.

Now let $N_{1}$ be any specific natural $N$ satisfying

$$
N_{1}>\max \left(N_{1}\left(\varepsilon_{1}\right), 2 A / \rho\right) \text {. }
$$

Next, we take $l \geq l_{1}\left(\varepsilon_{1}\right)$ sufficiently large s.t. in addition we also have $s_{(l-2) N_{1}}<$ $A / N_{1}$. It will be convenient to add the trivial restriction $l \geq 4$. Now, putting all these conditions together, take a specific $l$ with these restrictions and denote it by $l_{1}$. Hence,

$$
s_{(l-2) N_{1}}<A / N_{1}, \quad \forall l \geq l_{1} \geq \max \left(l_{1}\left(\varepsilon_{1}\right), 4\right) .
$$

Again, from now on whenever $N_{1}, l_{1}$ are mentioned, they will mean these specific natural numbers. After making all these preliminaries, we are able to present our key result in this paper.

Theorem 4.1. Let $\rho, A, \varepsilon_{1}, N_{1}, L_{1}$ be the numbers defined above. In addition let $N_{k}=2 N_{k-1}=2^{k-1} N_{1}$ for any natural $k$. Also, consider the functions $\left\{f_{l_{1} N_{k}}\right\},\left\{g_{l_{1} N_{k}}\right\},\left\{\varphi_{l_{1} N_{k}}\right\}$ as we have defined them. $\varphi_{l_{1} N_{k}}$ maps $\mathrm{HCP}_{l_{1} N_{k}}$ onto $\mathrm{HCP}_{l_{1} N_{k}}^{* *}$ and satisfies (3.2) for $N=N_{k}, l=l_{1}$. Then the radii of the circles in $\mathrm{HCP}_{2 N_{k}}^{* *}$ must satisfy the " $\rho$ condition" (2.1).

Proof. Before turning to the proof we point out two facts. First, it is enough to consider the " $\rho$ condition" only for the border circles of a given configuration. Indeed, it is quite obvious that the maximum principle holds for a discrete subharmonic function. Hence, if we apply this to the functions $r, 1 / r$ [1] we 
get that the "circle function" $r$ attains both its max and min value on the set of border circles. Thus, if $(2.1)$ is satisfied for the $m$ th generation of a configuration $\mathrm{HCP}_{m}^{\prime}$, it is satisfied for all generations of lower order, i.e., for all radii of circles appearing in $\mathrm{HCP}_{m}^{\prime}$.

The other fact is a simple (but useful) observation concerning a certain invariance property mentioned already in $\S 2$.

If we consider a certain configuration, say, $\mathrm{HCP}_{m}^{\prime}$, for some natural $m$, and "expand" it by a constant $\lambda>0$, then, for the new configuration $\lambda \times \mathrm{HCP}_{m}^{\prime}$, the ratio $r_{j} / r_{k}$ is transformed to $\lambda r_{j} / \lambda r_{k}$ which is the same. Hence, any statement about $s_{m}$, which is verified for $\mathrm{HCP}_{m}^{\prime}$, is also verified for $\lambda \times \mathrm{HCP}_{m}^{\prime}$. Thus, given arbitrary sequence $\left\{f_{l N}\right\}$ constructed as above, we may consider instead the sequence $\left\{\varphi_{l N}\right\}$ w.l.o.g.

We now turn to the proof, which will be by induction. First, consider the case $k=1$. With $N=N_{1},(4.2)$ takes the form

$$
\sum_{j=N_{1}+1}^{2 N_{1}} \sum_{l=1}^{6 j}\left(1-r_{j l}\right)^{2}<\varepsilon_{1}^{2} N_{1}^{2} .
$$

Consider, now, the configuration $\mathrm{HCP}_{l_{1} N_{1}}^{* *}$ and its subconfiguration $\mathrm{HCP}_{2 N_{1}}^{* *}$. By our choice of $l_{1}$ we have the validity of (4.4). Our aim is to show that the " $\rho$ condition" (2.1) is satisfied for the radii of the border circles of $\mathrm{HCP}_{2 N_{1}}^{* *}$. Assume the contrary, namely, that for some circle $K_{p, 2 N_{1}}$, which is a border circle of $\mathrm{HCP}_{2 N_{1}}^{* *}$, at least one of the following holds: either

$$
r_{p, 2 N_{1}} \leq e^{-3 \rho}
$$

or

$$
r_{p, 2 N_{1}} \geq e^{3 \rho} .
$$

We now show that each of the two assumptions (4.6), (4.6) leads to a contradiction. For this purpose, first observe that

$$
r_{j} / r_{j}^{\prime}<1+A / N_{1}
$$

for each two neighboring circles of $\mathrm{HCP}_{2 N_{1}}^{* *}$. Indeed, since $\mathrm{HCP}_{2 N_{1}}^{* *}$ is a subconfiguration of $\mathrm{HCP}_{l_{1} N_{1}}^{* *}$, each circle of $\mathrm{HCP}_{2 N_{1}}^{* *}$ is surrounded by at least $\left(l_{1}-2\right) N_{1}$ generations, which establishes (4.4), and thus $r_{j} / r_{j}^{\prime}<1+$ $s_{\left(l_{1}-2\right) N_{1}}<1+A / N_{1}$ which is $(4.7)$.

Next, choose a natural $m$ satisfying

$$
\rho / 2 A \leq m / N_{1} \leq \rho / A \leq 1 .
$$

Such an $m$ actually exists since by (4.3) $\rho / A-\rho / 2 A=\rho / 2 A>1 / N_{1}$ (also, $\rho \leq A(2.3)$ ). So now, make the assumption (4.6) for some circle $K_{p, 2 N_{1}}$ which is a border circle of $\mathrm{HCP}_{2 N_{1}}^{* *}$. Using (4.7) successively, we now show that for all circles that are not "too far" from $K_{p, 2 N_{1}}$ the weaker condition $r_{j} \leq e^{-\rho}$ follows. Indeed, take different "walks" starting at the "base" $K_{p, 2 N_{1}}$. Each "walk" is of at most $2 m$ "steps". For each circle of radius $r_{j}$ appearing in such a "walk" we have

$$
r_{j} \leq\left(1+\frac{A}{N_{1}}\right)^{2 m} r_{p, 2 N_{1}} \leq\left(1+\frac{A}{N_{1}}\right)^{2 m} e^{-3 \rho}<e^{2 m A / N_{1}-3 \rho} \leq e^{2 \rho-3 \rho}=e^{-\rho}
$$




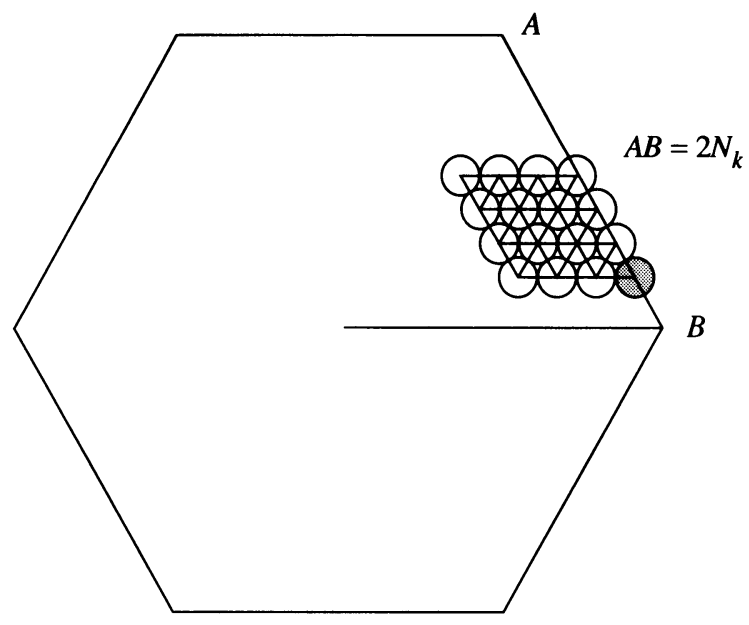

FIGURE 2

where we have used (4.6), (4.7) and (4.8). Since $m \leq N_{1}$ by (4.8), it follows that among such circles there is a rhombus containing $m^{2}$ circles arising from different "walks" starting in the "base" $K_{p, 2 N_{1}}$ and of at most $2 m$ "steps" (see Figure 2, where the case $m=4$ is illustrated. Note also that $2 m \leq 2 N_{1} \leq 2 N_{k}$ ). On the other hand, we get from (4.5) by summation on the $m^{2}$ circles:

$$
\varepsilon_{1} N_{1}^{2}>\sum_{N_{1}+1}^{2 N_{1}} \sum_{1}^{6 j}\left(1-r_{j k}\right)^{2}>m^{2}\left(1-e^{-\rho}\right)^{2}=m^{2}\left(e^{\rho}-1\right)^{2} e^{-2 \rho}>m^{2} \rho^{2} / 4 \text {. }
$$

The last inequality follows from (2.3). Using this, combined with (4.8) we get $\varepsilon_{1}^{2}>\left(m / N_{1}\right)^{2} \rho^{2} / 4 \geq \rho^{4} / 16 A^{2}$. But $\varepsilon_{1}=\rho^{2} / 4 A$ by (4.1) and this leads to a contradiction to the assumption (4.6). Assuming now (4.6)' instead of (4.6), and choosing $m$ by a similar procedure, we have

$$
\begin{aligned}
r_{j} & >r_{p, 2 N_{1}}\left(1+A / N_{1}\right)^{-2 m} \geq e^{3 \rho}\left(1+A / N_{1}\right)^{-2 m} \\
& >e^{3 \rho} e^{-2 m A / N_{1}} \geq e^{3 \rho-2 \rho}=e^{\rho} .
\end{aligned}
$$

Hence, $\varepsilon_{1}^{2} N_{1}^{2}>m^{2}\left(e^{\rho}-1\right)^{2}>\rho^{2} m^{2}$. It follows that

$$
\rho^{4} / 16 A^{2}=\varepsilon_{1}^{2}>\rho^{2} m^{2} / N_{1}^{2} \geq \rho^{4} / 4 A^{2},
$$

which is, again, a contradiction. Hence, the treatment of the case $k=1$ is complete.

We proceed, now, with the induction proof. So, assume that our statement has been established for $N_{k}$. If $\varphi_{l_{1} N_{k}}$ maps $\mathrm{HCP}_{l_{1} N_{k}}$ on $\mathrm{HCP}_{l_{1} N_{k}}^{* *}$, the induction assumption is that the " $\rho$ condition" is fulfilled for $\mathrm{HCP}_{2 N_{k}}^{* *}$.

Using Theorem 2.1, we have from (2.2) for $N=2 N_{k}$

$$
S_{l_{1} N_{k}} \leq \bar{S}_{2 N_{k}}<A / 2 N_{k}=A / N_{k+1} .
$$

Consider now $\varphi_{l_{1} N_{k+1}}$ that maps $\mathrm{HCP}_{l_{1} N_{k+1}}$ onto $\mathrm{HCP}_{l_{1} N_{k+1}}^{* *}$. For the subconfiguration $\mathrm{HCP}_{2 N_{k+1}}$ each border circle may be considered as the zero generation for a packing with $l_{1} N_{k+1}-2 N_{k+1}=\left(l_{1}-2\right) N_{k+1}$ generations. Using $l_{1} \geq 4$ (from (4.4)) and $N_{k+1}=2 N_{k}$, it follows that $l_{1} N_{k+1}-2 N_{k+1} \geq l_{1} N_{k}$. Hence, 
at least $l_{1} N_{k}$ generations surround each of the circles that belong to $\mathrm{HCP}_{2 N_{k+1}}$. Thus, from (4.9),

$$
r_{j} / r_{j}^{\prime}<1+A / N_{k+1}
$$

for each two neighboring circles of $\mathrm{HCP}_{2 N_{k+1}}^{* *}$. Also, by substituting $N=$ $N_{k+1} \geq N_{1}$ in (4.2)

$$
\sum_{j=N_{k+1}}^{2 N_{k+1}} \sum_{l=1}^{6 j}\left(1-r_{j l}\right)^{2}<\varepsilon_{1}^{2} N_{k+1}^{2} .
$$

The analysis now is identical with the previous treatment for $N_{1}$. Indeed, (4.11) replaces (4.5) and (4.10) replaces (4.7), and thus, the " $\rho$ condition" is established for $N_{k+1}$ which completes the induction process and the proof of the theorem.

\section{Proof of the Rodin Sullivan conjecture}

The RS conjecture [6] asserts that $s_{n}=O(1 / n)$ for $n \rightarrow \infty$. As proved by Rodin in [5] (see also [6]) this result implies the uniform convergence of the "circle function" $\left(r_{\varepsilon}\right.$, in Rodin's notation) to the modulus of the derivative of the Riemann map.

It is obviously enough to prove that $s_{n}<B / n$ for $n \geq n_{0}$ where $B$ is an absolute constant, and $n_{0}$ is some fixed natural number. Hence, it will be enough to prove

Theorem 5.1. Let $A, l_{1}, N_{1}$, be the numerical constants defined above. Then

$$
s_{n}<l_{1} A / n, \quad \forall n \geq l_{1} N_{1} .
$$

Proof. The proof is an easy consequence of Theorem 4.1 combined with Theorem 2.1.

We first consider the case $n=l_{1} N_{k}$ and then treat the general case $l_{1} N_{k} \leq$ $n \leq l_{1} N_{k+1}$. So, let $n=l_{1} N_{k}, N_{k}=2^{k-1} N_{1}$ where $k$ is an arbitrary natural number.

Also, consider any hexagonal packing $\mathrm{HCP}_{l_{1} N_{k}}^{\prime}$ which is combinatorially equivalent to $\mathrm{HCP}_{l_{1} N_{k}}$. Using the invariance property discussed previously, we may replace w.l.o.g. $\mathrm{HCP}_{l_{1} N_{k}}^{\prime}$ by the corresponding $\mathrm{HCP}_{l_{1} N_{k}}^{* *}$. From Theorem 4.1 we conclude that the radii of the circles in the subconfiguration $\mathrm{HCP}_{2 N_{k}}^{* *}$ must satisfy the " $\rho$ condition" (2.1). Now, using Theorem 2.1 applied for $\mathrm{HCP}_{2 N_{k}}^{* *} \subset \mathrm{HCP}_{l_{1} N_{k}}^{* *}$ we have from (2.2)

$$
S_{l_{1} N_{k}} \leq \bar{S}_{2 N_{k}}<A / 2 N_{k}=\left(l_{1} A / 2\right) / l_{1} N_{k},
$$

which implies (5.1) for $n=l_{1} N_{k}$, even with a better constant. Next, take any $n$ s.t. $n \geq l_{1} N_{1}$. Then, for some natural $k \geq 1$,

$$
l_{1} N_{k} \leq n \leq l_{1} N_{k+1}=2 l_{1} N_{k} .
$$

Hence, from (5.2), (5.3)

$$
s_{n} \leq s_{l_{1} N_{k}}<\left(l_{1} A / 2\right) / l_{1} N_{k}=l_{1} A / 2 l_{1} N_{k} \leq l_{1} A / n,
$$

which is the statement of Theorem 5.1. 


\section{ACKNOWLEDGMENT}

This research was supported by the fund for the promotion of research at the Technion.

\section{REFERENCES}

1. D. Aharonov, The hexagonal packing lemma and discrete potential theory, Canad. Math. Bull. 33 (1990), 277-252.

2. Z-Xu He, An estimate for hexagonal circle packings, J. Differential Geom. 33 (1991), 395-412.

3. O. Lehto and K. I. Virtanen, Quasiconformal mappings in the plane, Springer-Verlag, New York, Heidelberg, and Berlin, 1973, $258 \mathrm{pp.}$

4. B. Rodin, Schwartz's Lemma for circle packings, Invent. Math. 89 (1987), 271-289.

5. __ Schwartz's Lemma for circle packings. II, J. Differential Geom. 30 (1989), 539-554.

6. B. Rodin and D. Sullivan, The convergence of circle packings to the Riemann mapping, J. Differential Geom. 26 (1987), 349-360.

Department of Mathematics, Technion-Israel Institute of Technology, Haifa 3200 , ISRAEL

E-mail address: mar26aa@technion.bitnet 